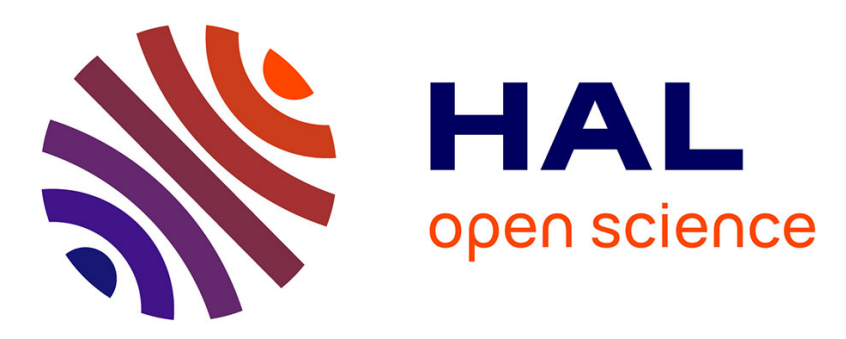

\title{
Validating Data from Semantic Web Providers
}

Jacques Chabin, Mírian Halfeld Ferrari Alves, Béatrice Markhoff, Thanh Binh

Nguyen

\section{To cite this version:}

Jacques Chabin, Mírian Halfeld Ferrari Alves, Béatrice Markhoff, Thanh Binh Nguyen. Validating Data from Semantic Web Providers. 44th International Conference on Current Trends in Theory and Practice of Computer Science (SOFSEM 2018), 2018, Krems, Australia. pp.682-695, 10.1007/978-3319-73117-9_48. hal-01836993

\section{HAL Id: hal-01836993 https://hal.science/hal-01836993}

Submitted on 18 Feb 2022

HAL is a multi-disciplinary open access archive for the deposit and dissemination of scientific research documents, whether they are published or not. The documents may come from teaching and research institutions in France or abroad, or from public or private research centers.
L'archive ouverte pluridisciplinaire HAL, est destinée au dépôt et à la diffusion de documents scientifiques de niveau recherche, publiés ou non, émanant des établissements d'enseignement et de recherche français ou étrangers, des laboratoires publics ou privés. 


\title{
Validating Data from Semantic Web Providers
}

\author{
Jacques Chabin ${ }^{1}$, Mirian Halfeld-Ferrari ${ }^{1}$, Béatrice Markhoff ${ }^{2}$, Thanh Binh \\ Nguyen $^{1 \star}$ \\ 1 Université d'Orléans, INSA CVL, LIFO, France \\ 2 Université Francois Rabelais de Tours, LI, France
}

\begin{abstract}
As the Linked Open Data and the number of semantic web data providers hugely increase, so does the critical importance of the following question: how to get usable results, in particular for data mining and data analysis tasks? We propose a query framework equiped with integrity constraints that the user wants to be verified on the results coming from semantic web data providers. We precise the syntax and semantics of those user quality constraints. We give algorithms for their dynamic verification during the query computation, we evaluate their performance with experimental results, and discuss related works.
\end{abstract}

Keywords: Semantic Web Data, User Quality Constraint, Query Rewriting

\section{Introduction}

There exist now very large knowledge bases on the web of Linked Open Data, as DBpedia, Yago or BabelNet. The largest ones contain millions of entities and billions of facts about them (attribute values and relationships with other entities) [20]. Applications are needed to help humans exploring this huge knowledge network, performing data analysis and data mining tasks. Promising recent proposals are currently experimented on only one semantic web data source $[7,10]$, and these processes can be expected to be even more helpful when they will deal with several linked open data sets. One crucial point for such applications, and in particular for data mining algorithms, is that the data collection and pre-processing steps have to be safe and sound.

In order to help semantic web data mining tool designers for performing the data collection and pre-processing steps, we propose a semantic web data validator [4]. The idea is to extend a query environment over semantic graph

* This work is supported by Girafon Project, funded by Region Centre Val de Loire. 
databases with a mechanism for filtering answers according to a user customized context. In this paper, we use the term "user" for the query-writer. The user context is composed of (i) the view she/he has defined on the needed semantic web data and (ii) a set of personalization tools, such as integrity constraints, confidence degrees, etc. In this paper, we only deal with integrity constraints, that we call user quality constraints, leaving the other kinds of personalization tools for other discussions (see $[4,6]$ ).

User quality constraints are restrictions imposed on query results. Both the constraints and the queries are expressed in terms of the user's view of data. The constraint verification is triggered by a query and consists in filtering its answers. In this way, there may be some inconsistencies within sources, but the answers given to the user are filtered to ensure their consistency w.r.t. her/his constraints. The following example illustrates the kind of constraints a user can define and what are their effects on query answers.

Example 1. Let us consider a query $q_{1}(X) \leftarrow$ teacher $O f(X, Y)$ in a context with two constraints:

$$
\begin{gathered}
c_{p}: \text { teacherOf }(X, Y) \rightarrow \text { professor }(X) . \\
c_{n}: \text { teacherOf }(X, Y), \text { takesCourse }(X, Y) \rightarrow \perp .
\end{gathered}
$$

The first constraint is to verify that each teacher of a course is a professor. The second constraint disallows to accept, in the query answers, a person who teaches a course while she/he is enrolled in the same course. Suppose the database is as in Figure 1. Although $\{B o b$, Tom, Alice, Ann $\}$ are answers to query $q_{1}$, $\{$ Alice, $A n n\}$ are invalid w.r.t. $c_{p}$, while $\{$ Tom $\}$ causes a violation of constraint $c_{n}$. Only $\{B o b\}$ satisfies all constraints. Thus, the answer to $q_{1}$ in the user context consisting of $\left\{c_{p}, c_{n}\right\}$ is $\{B o b\}$.

From Example 1, it can be noticed that when a constraint is triggered by instantiated atoms in the query's body, it requires auxiliary appropriate queries to verify its side effect. For instance, the fact teacherOf $(B o b, D B)$ triggers both $c_{p}$ and $c_{n}$, thus queries like $q_{11}() \leftarrow$ professor $(B \circ b)$ and

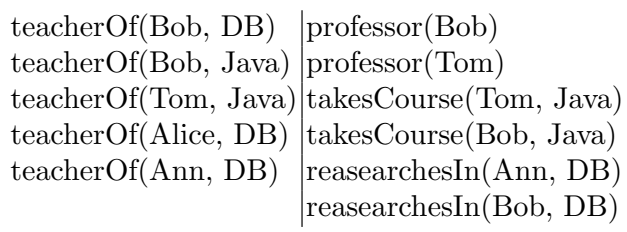

Fig. 1: Database instance $q_{12}() \leftarrow$ takesCourse $(B o b, D B)$ are produced to verify whether Bob is a professor and whether Bob is registered in the Database course. It is easy to see that, when dealing with a big amount of data, the impact of such auxiliary queries may be important. Even though most of them are simple queries, they can lead to a system overloading. A solution to avoid such issue is to integrate as much as possible the constraints into the query, in such a way that the answers will not only satisfy the initial query, but they will also respect all integrated constraints.

This paper is organized as follows: in Section 2, we present the overall query framework with user context, and precise the syntax and semantics of user quality constraints. In Section 3 we give algorithms for their dynamic verification during the query computation. In Section 4 we evaluate their performance with experimental results, and discuss related works. 


\section{A Querying Framework with Constraints}

\subsection{Querying environment}

Our query processing framework is depicted in Figure 2. It comprises two distinct parts which communicate: Data validation, responsible for checking constraints satisfaction, and Data providers for computing answers to the queries issued from the data validation part. The later may actually integrate several end-data-providers, or it may connect only one provider. For ensuring that the final answers to the user's queries satisfy all user constraints, a dialogue between the two parts is established, for getting intermediate results and sending subsidiary queries.

The user defines her/his context by setting her/his view on the queried sources, a set of datalog

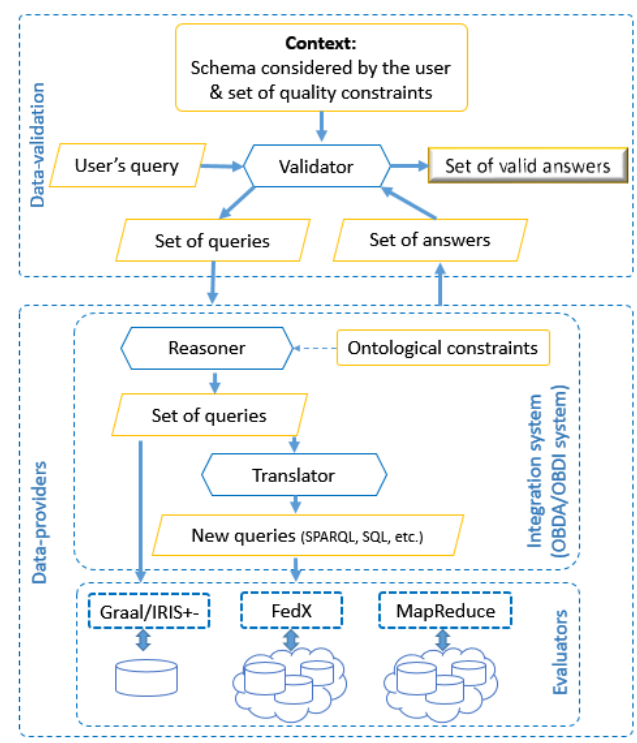

Fig. 2: Query system overview predicates as explained in next sec-

tion, and a set of quality constraints involving these predicates. The user's query involves these predicates, so quality constraints can be used as rewriting-rules to reformulate each query $q$, resulting in a union of conjunctive queries whose answers, contained in q's answers, are valid w.r.t. the user quality constraints.

Afterwards, these conjunctive queries are sent to the Data providers part, which evaluates them against data stored on sources. The query evaluation process is transparent to the validation step, in particular, answers that are entailed are treated in the same way as those that actually exist in sources. We respect the potential ontological dimension of semantic web sources, while interpreting the user constraints using the closed-world assumption. Indeed, as it deals with semantic data, the evaluating process performed by the Data providers part relies on the open-world assumption, where ontological constraints are used to deduce new information. Ontological constraints are used as rewriting-rules to reformulate a query into a set of new conjunctive queries, for taking into account integration information (OBDA/OBDI Systems $[18,3]$ ), or for dealing with incomplete information issues $[3,11,15,12]$. But such rewritings are performed by the Data providers part, independently from the Data validation part.

As our system may be deployed with various data management systems, a module will translate datalog+- queries [5] (used by $\mathrm{Graal}^{3}$ ) into SPARQL for FedX [19], and HIVE-SQL for MapReduce (as proposed in [4]).

\footnotetext{
${ }^{3}$ https://graphik-team.github.io/graal/
} 


\subsection{Constraints}

Our constraints are expressed in a first-order logic formalism. We consider an alphabet made up of three disjoint sets const, var and pred, of constants, variables and predicate names, respectively. A term $t$ is either a variable or a constant and an atom is a formula $p\left(x_{1}, x_{2}, \ldots, x_{n}\right)$ where $p$ is a predicate name and each $x_{i}$ is a term. A substitution is a total mapping $\sigma:$ var $\rightarrow T$ from variables to terms. A homomorphism from a set of atoms $A_{1}$ to a set of atoms $A_{2}$, all over the same schema $R$, is a substitution $h$ from the set of terms of $A_{1}$ to the set of terms of $A_{2}$ such that: (i) if $t$ is a constant, then $h(t)=t$, and (ii) if $r\left(t_{1}, \ldots, t_{n}\right)$ is in $A_{1}$, then $h\left(r\left(t_{1}, \ldots, t_{n}\right)\right)=r\left(h\left(t_{1}\right), \ldots, h\left(t_{n}\right)\right)$ is in $A_{2}$. The notion of homomorphism naturally extends to conjunctions of atoms. Two atoms $A_{1}$ and $A_{2}$ are unifiable if and only if there exists a substitution $\sigma$ s.t. $\sigma\left(A_{1}\right)=\sigma\left(A_{2}\right)$. Furthermore, if two atoms $A_{1}$ and $A_{2}$ are unifiable then there exists a most general unifier (mgu) $\theta$ s.t. $\theta\left(A_{1}\right)=\theta\left(A_{2}\right)$.

A conjunctive query (CQ) $q$ of arity $n$ over a given schema is a logical rule of the form $q(\mathbf{X}) \leftarrow \phi(\mathbf{X}, \mathbf{Y})$, where $\phi(\mathbf{X}, \mathbf{Y})$ is a conjunction of atoms over the schema, $q$ is a n-ary predicate and $\mathbf{X}, \mathbf{Y}$ are sequences of terms. Given a logical rule $r$, we denote by $\operatorname{body}(r)$ the rule's antecedent by head $(r)$ its consequent.

Our user quality constraints [4] are also logical rules. We define a user context as a set $\mathcal{C}$ of constraints, composed of three subsets, as follows:

Positive constraints $\left(\mathcal{C}_{P}\right)$ : Each positive constraint has the form

$$
\forall \mathbf{X}, \mathbf{Y} \quad L_{1}(\mathbf{X}, \mathbf{Y}) \rightarrow \exists \mathbf{Z} \quad L_{2}(\mathbf{X}, \mathbf{Z})
$$

$L_{1}(\mathbf{X}, \mathbf{Y})$ and $L_{2}(\mathbf{X}, \mathbf{Z})$ are atoms and $\mathbf{Z}$ are existential variables.

Negative constraints $\left(\mathcal{C}_{N}\right)$ : each negative constraint is a rule having the form $\forall \mathbf{X} \phi(\mathbf{X}) \rightarrow \perp$

where $\phi(\mathbf{X})$ is an atom $L_{1}(\mathbf{X})$ or a conjunction of two atoms $L_{1}\left(\mathbf{X}_{1}\right), L_{2}\left(\mathbf{X}_{2}\right)$, which have a non-empty intersection between the terms in $\mathbf{X}_{1}$ and $\mathbf{X}_{2}$. We refer to $\mathcal{C}_{N 1}$ and $\mathcal{C}_{N 2}$ as sets of negative constraints having only one atom and two atoms, respectively. Negative constraint is a special case of denial constraint with at most two occurrences of database literals.

Equality-generating dependency constraints without nulls $\left(\mathcal{C}_{K}\right)$ : each EGD is a rule having the general form

$$
\forall X_{1}, X_{2}, \mathbf{Y}, \mathbf{Z}_{1}, \mathbf{Z}_{2} L_{1}\left(\mathbf{Y}, X_{1}, \mathbf{Z}_{1}\right), L_{2}\left(\mathbf{Y}, X_{2}, \mathbf{Z}_{2}\right) \rightarrow X_{1}=X_{2} .
$$

where $\mathbf{Y}$ is a sequence of common terms of $L_{1}$ and $L_{2}$ that has at least one element. Notice that EGD include functional dependency (and thus, key constraints) having the form $L_{1}\left(\mathbf{Y}, X_{1}, \mathbf{Z}_{1}\right), L_{1}\left(\mathbf{Y}, X_{2}, \mathbf{Z}_{2}\right) \rightarrow X_{1}=X_{2}$.

In the rest of this paper, for simplicity, we will omit the quantifiers. We say that a constraint $c$ is triggered by an atom $A$ when there is a homomorphism $h$ from $\operatorname{body}(c)$ to $A$. Positive constraints are a special case of linear tuple generating dependency (TGD [2]) which contain only one atom in the head. They cover both inclusion dependency class and join dependency class [2]. When $\mathbf{Z}$ is not empty, the homomorphism $h$ is extended to $h^{\prime}$ such that, for each existential variable $z \in \mathbf{Z}, h^{\prime}(z)$ is a new fresh variable. It is well-known that facts from a database instance may trigger such constraints, and the chase procedure ([17]) is the standard process for the generation of new facts from a database instance 
and a set of dependencies (TGD or EGD) [2]. It can also be used to decide containment of conjunctive queries in the presence of constraints ([14]). In this paper, we consider that the set of positive constraints is weakly acyclic, which guarantees the decidability of query containment [9]. In Example 1, $c_{p}$ and $c_{n}$ are illustrations for the definitions of positive and negative constraints above. An example of EGD constraints can be as follows:

$$
c_{k}: \text { worksFor }(X, Y, Z) \text {, headOf }(X, W) \rightarrow Z=W .
$$

It states that if a person $\mathrm{X}$ is the head of $\mathrm{W}$ and if she is working for organization $\mathrm{Y}$ in department $\mathrm{Z}$ then $\mathrm{W}$ must be the department $\mathrm{Z}$.

\section{$3 \quad$ Validating Semantic Web Query Outputs}

Given a user's query $q$, the validation of its result on the basis of user's quality constraints in $\mathcal{C}$ can be performed in two ways: by rewriting $q$ to take into account the constraints in $\mathcal{C}$, or by the evaluation of auxiliary queries, composed on the basis of initial results obtained for $q$. Even if the choice between these two processes depends on the query evaluation power of data providers, it is important to study their costs and benefits in a common framework. To do so, in this paper, we use Graal [1] as conjunctive query evaluator for both techniques. More precisely, we focus on testing and comparing the performance of our validation approach in the following two scenarios: (1) the rewriting of $q$ on the basis of constraints in $\mathcal{C}$, followed by the rewritten-query evaluation, and (2) what we call the naive solution, i.e. evaluate $q$, then build and evaluate multiple auxiliary queries on the basis of $q$ 's answers. This section summarizes these two scenarios, and in Section 4 we analyse in details their respective validation performance.

\subsection{Query Rewriting with Constraints}

Given a CQ $q$ and a set of constraints $\mathcal{C}$, let us consider examples to illustrate the situations our query rewriting algorithm tackles with.

Example 2. Query $q_{1}$ below looks for professors who were born in a foreign country. Constraints establish a user's context imposing a professor to be associated with a course $\left(c_{p_{a}}\right)$ offered by a department $\left(c_{p_{b}}\right)$. Moreover, the user is interested only in professors working in the public sector $\left(c_{p_{c}}\right)$.

$$
\begin{gathered}
q_{1}\left(X_{1}\right) \leftarrow \text { professor }\left(X_{1}\right), \text { placeOfBirth }\left(X_{1}, Z_{1}\right), \text { foreignCountry }\left(Z_{1}\right) . \\
c_{p_{a}}: \operatorname{professor}(X) \rightarrow \text { teacherOf }(X, Y) . \\
c_{p_{b}}: \text { teacherOf }(X, Y) \rightarrow \text { offeredCourseIn }(Y, Z) . \\
c_{p_{c}}: \operatorname{professor}(X) \rightarrow \text { employeeGov }(X) .
\end{gathered}
$$

In this context, we see body $\left(q_{1}\right)$ as a set of atoms capable of triggering constraints and producing new atoms that should be added to the query's body. This operation corresponds to a chase computation ([17]), which starts with the atoms in $\operatorname{body}\left(q_{1}\right)$. Special attention is required in the use of variable renaming. The new rewritten query, that the system should send to data providers, is: $q_{1}^{\prime}\left(X_{1}\right) \leftarrow$ professor $\left(X_{1}\right)$, teacherOf $\left(X_{1}, Y_{1}\right)$, of feredCourseIn $\left(Y_{1}, Y_{2}\right)$, employeeGov $\left(X_{1}\right)$, placeOfBirth $\left(X_{1}, Z_{1}\right)$, foreignCountry $\left(Z_{1}\right)$. 
When the query, or the constraints, contain constants, the above rewriting technique should be revised, as illustrated by the following example.

Example 3. Consider query $q_{2}$, and constraint $c_{p 2}$ imposing restrictions on database teachers - they should do research in the database domain:

$$
\begin{gathered}
q_{2}(X) \leftarrow \text { teacherOf }(X, Y) . \\
c_{p 2}: \text { teacherOf }(Z, D B) \rightarrow \text { researchesIn }(Z, D B) .
\end{gathered}
$$

Notice that no restriction is imposed on teachers in other domains. Here we cannot apply the chase as in Example 2, because a query $q_{2}^{\prime}(X) \leftarrow$ teacher $O f(X, D B)$, researchesIn $(Z, D B)$ would ignore the teachers of all other domains. In this case, our proposal is to replace $q_{2}$ by the union of the two following queries:

$$
q_{2.1}(X) \leftarrow \text { teacherOf }(X, Y), \neg \text { teacherOf }(X, D B) \text {. }
$$

$q_{2.2}(X) \leftarrow$ teacherOf $(X, Y)$, teacherOf $(X, D B)$, researchesIn $(X, D B)$.

Algorithm 1 summarizes our rewriting solution. In this algorithm the input is composed of a conjunctive query, and positive and negative constraints. However, negative constraints in $\mathcal{C}_{N 2}$, i.e., those having the form $L_{1}\left(\mathbf{X}_{1}\right), L_{2}\left(\mathbf{X}_{2}\right) \rightarrow \perp$ are transformed into two equivalent formulas: $L_{1}\left(\mathbf{X}_{1}\right) \rightarrow \neg L_{2}\left(\mathbf{X}_{2}\right)$ and $L_{2}\left(\mathbf{X}_{2}\right) \rightarrow$ $\neg L_{1}\left(\mathbf{X}_{1}\right)$. In this way, negative constraints receive a similar treatment as positive constraints. For instance, from Example 1, the constraint $c_{n}$ can be written as $c_{n_{1}}$ : teacherOf $(X, Y) \rightarrow \neg$ takesCourse $(X, Y)$ and $c_{n_{2}}$ :takesCourse $(X, Y) \rightarrow$ $\neg$ teacher $O f(X, Y)$. Query $q_{1}$ is then rewritten as $q_{1}(X) \leftarrow \operatorname{teacher} O f(X, Y)$, $\neg$ takesCourse $(X, Y)$.

In Algorithm 1, Function RewriteWithConstraints is the main program, which ensures that each query is rewritten by taking into account all positive and negative constraints in $\mathcal{C}$. It calls Function Integrate, the kernel of our rewriting method, which computes the new queries that replace the given query $q$, by integrating in $q$ the restrictions imposed by the given constraint $c$.

The instantiation of constraints w.r.t. the atoms $L$ in $q$ 's body is done on line 15 by using a $m g u \theta$, and $c^{\prime}$ is the resulting constraint, instantiated with constants in $q$. Then, on line 18, we consider the cases where $c^{\prime}$ can be triggered by $L$. This happens when $\theta$ is a variable renaming, or, when it replaces variable in $c$ by constants in $L$ (afterwards, there may still exists a homomorphism $\nu$ from body $\left(c^{\prime}\right)$ to $\left.L\right)$. For instance, consider query $q_{3}$ and constraint $c_{3}$ as follows:

$$
\begin{gathered}
q_{3}(X) \leftarrow \text { professor }(B o b), \text { teacherOf }(B o b, X) \\
c_{3}: \text { professor }(X) \rightarrow \operatorname{inDept}(X, Y)
\end{gathered}
$$

With $L=\operatorname{professor}(B o b)$ and $\theta=\{X / B o b\}$, we obtain $c_{3}^{\prime}: \operatorname{professor}(B o b) \rightarrow$ $\operatorname{inDept}\left(B o b, Y_{1}\right)$, where $Y_{1}$ is a new variable resulting from variable renaming performed by createRule (line 19). Similarly, in Example 2, for $L=\operatorname{professor}\left(X_{1}\right)$ and $\theta=\left\{X / X_{1}\right\}$ we obtain $c_{p_{a}}^{\prime}$ : professor $\left(X_{1}\right) \rightarrow$ teacherOf $\left(X_{1}, Y_{1}\right)$.

When the homomorphism $\nu$ exists, the query's body is completed with the head of $c^{\prime}$ (line 19). The loop on line 5 ensures that the query's body will be completed with all the atoms obtained by triggered constraints. Notice that the idea here is to use a chase procedure applied to rules that respect some syntactic restrictions. Indeed, our current implementation deals with a set of weakly acyclic TGD ([9]). Roughly, a set of TGD is acyclic if it does not allow 
for cascading of labelled null creation during the chase. Example 2 illustrates a rewritten query obtained by following the above steps.

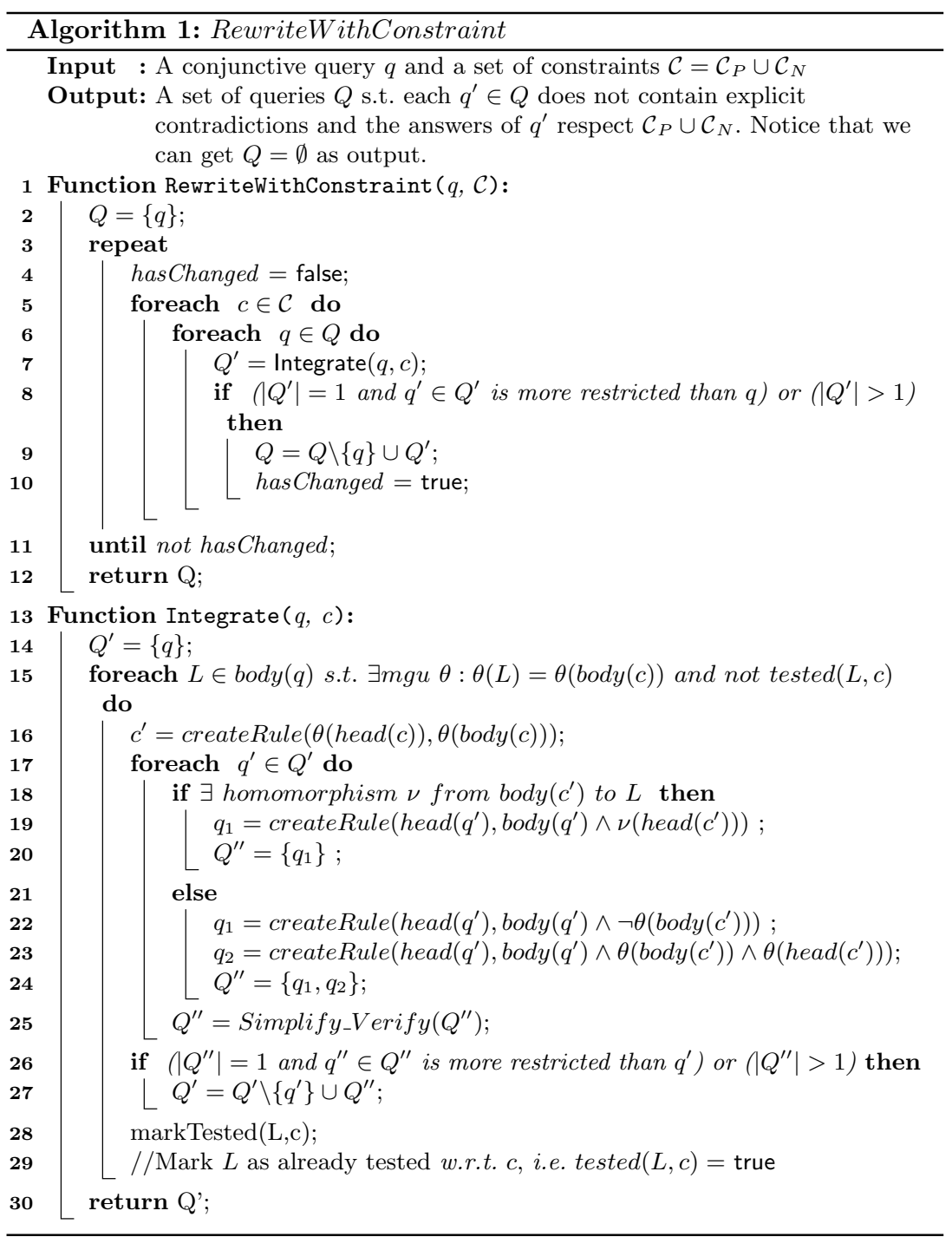

When the homomorphism $\nu$ does not exist, we are dealing with constants that cannot map to variables or with different constants. Let us consider Example 3, after executing line 15 of Algorithm 1 with $L=$ teacherOf $(X, Y)$. We have $c_{p 2}^{\prime}:$ teacherOf $(Z, D B) \rightarrow$ researchesIn $(Z, D B)$ (no changes w.r.t. $c_{p 2}$ ). No homomorphism from body $\left(c^{\prime}\right)$ to $L$ is possible. Line 22 deals with results that are not concerned by the constraint. In this case, the query body is completed with the negation of the constraint's body. Thus, in our Example 3, $q_{2.1}$ selects people who do not teach $D B$. With the database instance of Figure 1, the answer for $q_{2.1}$ is Tom. Then, on line 23, we deal with results concerned by the constraint. In 
Example 3, $q_{2.2}$ selects two kinds of people: $(i)$ those who are database researchers and only teach $D B$ and $(i i)$ those who teach and do research in the database domain but also teach other subjects. Continuing with our example, the desired answers for $q_{2}$ are Bob, Ann and Tom. With our algorithm, Bob and Ann are not answers for $q_{2.1}$, but they are answers to $q_{2.2}$. The result of $q_{2}$ is the union of the answers for $q_{2.1}$ and $q_{2.2}$.

Rewritten queries, put in the set $Q^{\prime \prime}$, are sent to function Simplify_Verify (line 25) that, for each query, removes redundant atoms. This function also ensures that $Q^{\prime \prime}$ does not contain queries with explicit contradiction. In other words, the function checks whether: $(i)$ there is no two atoms having the form $L(\mathbf{X})$ and $\neg L(\mathbf{X})$ in the query body and $(i i)$ atoms in the query body cannot trigger a negative constraint.

We use query containment (see, for instance [2] for a revision on the subject) to decide whether a rewritten query replaces a given one. On line 27 , notice that at each iteration step, the set $Q^{\prime}$ contains the most restricted rewritten queries obtained so far. Each iteration step considers an atom in the query body and one single constraint. The output of the Integrate function is the set $Q^{\prime}$, which contains the most restricted rewritten queries obtained for one query w.r.t. one constraint $c$. Then, on line 9 , the replacement of the original query $q$ is considered. If only one query $q^{\prime}$ results from Integrate, $q$ is replaced by $q^{\prime}$ only when $q^{\prime}$ is more restricted than $q$. Otherwise, when more than one rewritten queries result from Integrate, $q$ is replaced by them.

The query obtained after only chasing the original query w.r.t. positive constraints corresponds to the universal plan of [8]. However, when dealing with negative constraints, even when Integrate performs only lines 17-20 to rewrite a given query, the rewritten query may contain negative atoms.

\subsection{Building Auxiliary Queries}

Given a query $q$, to ensure its answer consistency w.r.t. user's quality constraints, instead of dealing with query rewriting, one can consider the generation of subqueries from the initial answers obtained from $q$. Let $h_{t}$ be the homomorphism used to produce tuple $t$ as an answer to the query $q$. We want to check whether $t$ is valid w.r.t. constraints. Tuple $t$ is considered valid only when all constraints triggered during the validation process are satisfied.

Let $L(\mathbf{X})$ be an atom of $\operatorname{body}(q)$. The instantiated atom $h_{t}(L(\mathbf{X}))$ may trigger a constraint $c$. According to the type of $c$, an auxiliary query $q^{\prime}$ is created:

- For $c \in \mathcal{C}_{P}$ the auxiliary boolean query is $q^{\prime}() \leftarrow h_{t}\left(L_{0}\left(\mathbf{X}_{0}\right)\right)$ where $L_{0}\left(\mathbf{X}_{0}\right)=$ head $(c)$. The resulting tuple $t$ is valid w.r.t. $c$ if the answer of $q^{\prime}$ is positive. Notice however that each fact $f$ resulting from the instantiation of $h_{t}\left(L_{0}\left(\mathbf{X}_{0}\right)\right)$ on the database may trigger another constraint. The validation process continues until no constraint is triggered and corresponds to a chase procedure, establishing a dialogue between the validator and the providers.

- For $c \in \mathcal{C}_{N}$ and assuming that $c$ has the form $L(\mathbf{X}), L_{0}\left(\mathbf{X}_{0}\right) \rightarrow \perp$ the auxiliary boolean query is $q^{\prime}() \leftarrow h_{t}\left(L_{0}\left(\mathbf{X}_{0}\right)\right)$. Tuple $t$ is valid w.r.t. $c$ if the 
answer of $q^{\prime}$ is negative. Clearly, if $c$ has the form $L(\mathbf{X}) \rightarrow \perp$, the verification is straightforward.

- For $c \in \mathcal{C}_{K}$, assuming that $c$ has form $L\left(\mathbf{Y}, X_{1}, \mathbf{Z}_{1}\right), L_{0}\left(\mathbf{Y}, X_{2}, \mathbf{Z}_{2}\right) \rightarrow X_{1}=$ $X_{2}$ and $\mathbf{X}=\mathbf{Y} \cup X_{1} \cup \mathbf{Z}_{1}$, the auxiliary query is $q^{\prime}\left(X_{2}\right) \leftarrow h_{t}\left(L_{0}\left(\mathbf{Y}, X_{2}\right.\right.$, $\left.\mathbf{Z}_{2}\right)$ ). Tuple $t$ is valid w.r.t. $c$ if the answer set is a singleton containing the tuple value $h_{t}\left(X_{1}\right)$.

\subsection{Complete Validation}

Finally, Algorithm 2 is responsible for validating the result of a query $q$ w.r.t. a set of constraints $\mathcal{C}$. Algorithm 1 rewrites the query only w.r.t. positive and negative constraints. Then it must be completed by the generation of auxiliary queries, from the answers of the rewritten queries, at least for dealing with EGD constraints in $\mathcal{C}$. On line 2 of Algorithm 2, Function RewriteWithConstraint returns a set $Q$ of rewritten queries. Afterwards, Function Eval evaluates all queries in $Q$ (line 3), and answers are stored in the set Solutions. On line 5, $\mathcal{C}_{\text {check }}$ is the set of the constraints which are not addressed by Algorithm 1. Function Valid verifies whether an answer sol is valid w.r.t. $\mathcal{C}_{\text {check }}$ by generating corresponding auxiliary queries, as sketched in Section 3.2.

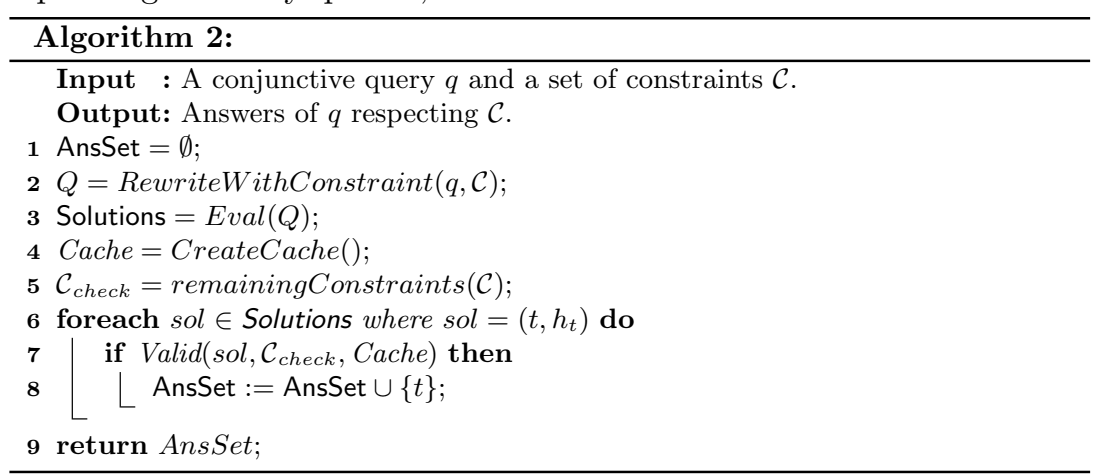

\section{Experimental Results and Related Works}

Our main goal is to compare the overall performance between $(i)$ our first scenario, i.e. the query rewriting approach performed by Algorithm 2 when only the EGD constraints are not considered by Function RewriteWithConstraint, and (ii) our second scenario, the naive approach, performed by Algorithm 2 when Function RewriteWithConstraint is simply not applied. Both approaches compute the same valid answers (whose number is given in column 5 and 6 in Table 1(a) for the given conjunctive query, i.e. answers that satisfy the given set of quality constraints. Another important goal of experiments is to analyze features that affect the computation efficiency, such as the size of datasets, the size of queries, the number and type of constraints, etc.

We performed experiments using a HP ZBook laptop equipped with a quadcore Intel $17-4800 \mathrm{MQ}$ processors at $2.7 \mathrm{GHz}$ and $16 \mathrm{~Gb}$ of RAM. We developed 


\begin{tabular}{|c|c|c|c|c|c|c|}
\hline \multirow{2}{*}{\multicolumn{2}{|c|}{$\begin{array}{l}\text { Trig } \\
\text { cons }\end{array}$}} & \multicolumn{2}{|c|}{ Num.Rew.Que. } & \multirow{2}{*}{$\begin{array}{l}\text { Max num. } \\
\text { atoms }\end{array}$} & \multicolumn{2}{|c|}{ Valid answers } \\
\hline & & w.opt. & wo.opt. & & 1 univ & 5 univ \\
\hline Q1 & & 1 & 4 & 7 & 523 & 3331 \\
\hline Q2 & 1 & 1 & 1 & 2 & 7861 & 36682 \\
\hline Q3 & 2 & 2 & 2 & 5 & 3599 & 23749 \\
\hline Q4 & 0 & 1 & 1 & 2 & 10735 & 67702 \\
\hline Q5 & 6 & 6 & 8 & 14 & 50 & 59 \\
\hline \begin{tabular}{|l|} 
Q6 \\
\end{tabular} & 8 & 2 & 8 & 13 & 6631 & 36538 \\
\hline Q7 & 6 & 2 & 8 & 13 & 21 & 220 \\
\hline
\end{tabular}

(a) Queries and Rewritten Queries

\begin{tabular}{|l|r|r|r|r|r|}
\cline { 2 - 7 } \multicolumn{1}{c|}{} & \multicolumn{2}{c|}{ 1 university } & \multicolumn{2}{c|}{5 universities } \\
\cline { 2 - 7 } \multicolumn{1}{c|}{} & RewTime & EvalTime & Total & EvalTime & Total \\
\hline Q1 & 0.043 & 0.372 & 0.415 & 0.492 & 0.535 \\
\hline Q2 & 0.001 & 0.429 & 0.430 & 6.388 & 6.389 \\
\hline Q3 & 0.007 & 0.124 & 0.131 & 0.804 & 0.811 \\
\hline Q4 & 0 & 0.111 & 0.111 & 0.692 & 0.692 \\
\hline Q5 & 0.048 & 0.702 & 0.75 & 0.773 & 0.821 \\
\hline Q6 & 0.011 & 20.522 & 20.533 & 122.285 & 122.296 \\
\hline Q7 & 0.01 & 3.193 & 3.203 & 162.105 & 162.115 \\
\hline
\end{tabular}

(b) Rewriting, Evaluation-Verification (s)

Table 1: Rewriting Approach

\begin{tabular}{|c|c|c|c|c|c|c|c|c|c|c|}
\cline { 2 - 12 } \multicolumn{1}{c|}{} & \multicolumn{4}{c|}{ 1 university } & \multicolumn{5}{c|}{5 universities } \\
\cline { 2 - 12 } \multicolumn{1}{c|}{ Eval. } & Verif. & Total & Init.ans. & Num.Que. & Eval. & Verif. & Total & Init.ans. & Num.Que. \\
\hline Q1 & 0.965 & 1.172 & 2.137 & 1548 & 2072 & 1.191 & 7.14 & 8.331 & 10095 & 13426 \\
\hline Q2 & 0.153 & 49.952 & 50.105 & 7861 & 7861 & 1.038 & t/o & t/o & 36682 & - \\
\hline Q3 & 0.041 & 1.515 & 1.556 & 3599 & 3599 & 2.59 & 10.709 & 13.299 & 23749 & 23749 \\
\hline Q4 & 0.026 & 0.072 & 0.098 & 10735 & 0 & 0.166 & 0.43 & 0.596 & 67702 & 0 \\
\hline Q5 & 0.227 & 1.704 & 1.931 & 50 & 200 & 0.735 & 1.363 & 2.098 & 59 & 236 \\
\hline Q6 & 9.205 & 57.948 & 67.153 & 6631 & 39786 & 16.108 & t/o & t/o & 36538 & - \\
\hline Q7 & 4.772 & 0.535 & 5.307 & 96 & 159 & 292.216 & 0.712 & 292.928 & 645 & 1305 \\
\hline
\end{tabular}

Table 2: Evaluation and Verification in the Naive Approach (s)

Java programs using Graal, a Java toolkit dedicated to knowledge-base querying within the framework of existential rules (e.g. Datalog+-). We used the LUBM $^{4}$ benchmark, which describes the organizational structure of universities with 43 classes and 32 properties, and provides a generator of synthetic data with varying size. For analyzing the impact of the size of databases on the tested solutions, we created two versions of datasets containing data of 1 and 5 universities, containing 86,165 and 515,064 triples, respectively. These datasets are loaded and managed directly by Graal, which converts them from RDF /XML to Dlgp, its supported data format. Inspired by the 14 test queries of LUBM, we devised 7 queries and 12 constraints written in Dlgp (4 positive, 5 negative, and 3 keys) ${ }^{5}$.The queries spread from simple queries with few atoms $(Q 1$, $Q 2)$ to more complex queries $(Q 6, Q 7)$, and may contain constants $(Q 5)$. Some constraints also involve constants $(C p 2, C p 3, C p 4)$. Column 1 in Table 1(a) contains the number of constraints triggered by each query. The second and third columns present the number of rewritten queries either applying the simplification query-containment test (Function Simplify_Verify), or not. Theoretically, a query that involves $n$ constraints can be rewritten into $2^{n}$ reformulations in the worst case. Experimental results show that in some cases $(Q 1, Q 6, Q 7)$, Function Simplify_Verify significantly reduces the number of rewritings. Column 4 shows the maximum number of atoms in rewritten queries, which demonstrates that the more constraints are used in the rewriting procedure, the more complex are the rewritings (number atoms or joins).

\footnotetext{
${ }^{4}$ Lehigh University: http://swat.cse.lehigh.edu/projects/lubm/

${ }^{5}$ Details in the techinical report: http://www.univ-orleans.fr/lifo/rapports. php?annee $=2017$
} 
We now turn our attention to the time of rewriting and complete evaluationverification, reported in Table 1(b), which contains the following information: (i) the time needed for rewriting, indicated in Column RewTime; (ii) the time needed for evaluating all queries obtained from the rewriting step, shown in column EvalTime, for the two tested datasets; (iii) the total time for performing these two steps (Column Total). Rewritings are very fast and the evaluation time is clearly the major part in the total time, in all cases. Furthermore, the evaluation time is directly proportional to the size of the tested dataset. Moreover, the rewritten-query complexity affects the evaluation time, for instance, $Q 6$ and $Q 7$ have 13 atoms in their body and their evaluation times on 5 universities are the biggest ones. Interestingly, $Q 5$ has 14 atoms and does not need so much time for the evaluation. The reason is that $Q 5$ contains a constant, which highly reduces its querying space. In summary, these first experiments demonstrate how the dataset size, the query complexity, the number of involved constraints and the presence of constants in initial and rewritten queries, impact the overall time of the rewriting-and-evaluating approach for processing a query with user-constraints.

Concerning now the experimental results for the naive approach, shown in Table 2, we have, for each dataset: $(i)$ the time needed for evaluating the initial query in Column Eval.; (ii) the time necessary for generating and executing auxiliary queries to verify all answers obtained from the previous evaluation step, in Column Verif.; (iii) the overall processing time in Column Total. (iv) the number of answers before constraint verification in Column Init.Ans.; and $(v)$ the number of auxiliary queries generated, in Column Num. Queries. Naturally, the dataset size has a similar effect as in the rewriting approach. However, the number of generated auxiliary queries plays an even more significant role in the total processing time. Intuitively, this number depends $(i)$ on the size of the initial answer set and (ii) on the number of involved constraints. We can notice that, contrary to the rewriting approach, the complexity of the query has little effects on the total execution time in the naive approach. See, for instance, $Q 6$ and $Q 7$ which have similar complexity. However, $Q 6$ has many answers, provoking the generation of many sub-queries. Indeed, the verification step is carried out by generating simple sub-queries for each answer w.r.t. each constraint.

Perhaps one of the most meaningful observation provided by our experiments is that, when the dataset size increases, the rewriting approach is clearly far more efficient than the naive approach. This is specially the case when the initial query gives a large number of answers, no matter if it is a simple or a complex query, and these answers trigger a lot of constraints: $Q 2$ and $Q 6$ are typical examples of such cases, which induce a time-out for 5 universities. For $Q 4$, which triggers no constraint, the naive approach is better or similar to the rewriting one.

Related Works We already mentioned the main works related to our proposal in Section 2.1. Firstly, ontological-constraints-based query-rewritings in Ontology-Based Data Access (OBDA) systems $[18,3]$ and rewritings in incomplete information querying systems $[11,15,12]$ inspired our solution. In [16] we also find different semantics for query answering over inconsistent Datalog $^{ \pm}$ 
ontologies. Their goal is to propose corrections to the database, while ours is to avoid answering on the basis of inconsistent data. Indeed, we designed our solution with traditional database constraints that must be verified, while in those works ontological constraints are seen as inference rules. Our user constraints allows us to verify answer sets and eleminate those answers that do not comply with the user needs. For instance, coming back to $c_{p}$ given in Introduction, which enforces that all person who teaches is a professor, the answer teacherOf $(B o b, D B)$ is valid only if professor $(B o b)$ is true in the provided answers, i.e. the fact professor $(B o b)$ is not inferred from the user constraints.

For this reason, our rewriting algorithm is based on traditional results in the database domain already cited in Section $2.2[2,17,14]$. We are currently studying to what extent our proposed user-context is covered by the traditional framework of answering queries using views, for which a general rewriting algorithm is presented in [8], and further improved in [13]. We already mentioned this algorithm, called C\&B for its two phases (Chase and BackChase), at the end of Section 3.1. It first constructs a canonical rewriting called UniversalPlan by using TGDs rules, which play the same role as our positive constraints, and then it searches minimal reformulations among the candidates in the UniversalPlan, using EGDs rules. But how it could apply to our context is not obvious, because we already mentioned that, in general, the Chase can not be directly used with constraints containing constants, excepted when there exists a homomorphism from the constraint's atoms to the query's atom (see Lines 17-20 in Algorithm 1).

\section{Conclusion}

We presented a solution for validating a set of user quality constraints when performing query evaluation, in the semantic web context. A naive way to verify them is to generate auxiliary queries after having got the result set from the evaluation of the user query. Our experiments have put in evidence that these auxiliary queries, generally simple but performed on huge data sets, sometimes lead to overload the system. Integrating as much as possible the constraints into the original user query can help to overcome this drawback. We presented an algorithm for such a constraint-query integration, and provided experimental results that demonstrate its benefits regarding total query-with-constraints processing time. Both techniques are correct and complete. In other words, given the query $Q$ and the constraints $C$, (i) there is no answer to $Q$ that satisfies $C$, but is not in the answer set of both methods (completeness); (ii) all the answers produced by both algorithms are answers to $Q$ that respect $C$ (correction). Our immediate future works will concern extending our experiments to take into account the data provider features and capabilities (e.g. not all of them can evaluate complex queries).

\section{References}

1. Graal. At https://graphik-team.github.io/graal/. 
2. S. Abiteboul, R. Hull, and V. Vianu. Foundations of databases, volume 8. AddisonWesley Reading, 1995.

3. S. Abiteboul, I. Manolescu, P. Rigaux, M.-C. Rousset, and P. Senellart. Web Data Management. Cambridge University Press, New York, NY, USA, 2011.

4. M. Bamha, J. Chabin, M. Halfeld-Ferrari, B. Markhoff, and T. B. Nguyen. Personalized environment for querying semantic knowledge graphs: a mapreduce solution. Technical report, LIFO- Université d'Orléans, RR-2017-06, 2017.

5. A. Calì, G. Gottlob, and T. Lukasiewicz. A general datalog-based framework for tractable query answering over ontologies. J. Web Sem., 14:57-83, 2012.

6. J. Chabin, M. Halfeld-Ferrari, and T. B. Nguyen. Querying Semantic Graph Databases in View of Constraints and Provenance. Technical report, LIFO- Université d'Orléans, RR-2016-02, 2016.

7. C. d'Amato, A. G. B. Tettamanzi, and T. D. Minh. Evolutionary discovery of multi-relational association rules from ontological knowledge bases. In $E K A W$, pages 113-128. Springer International Publishing, 2016.

8. A. Deutsch, L. Popa, and V. Tannen. Query reformulation with constraints. SIGMOD Rec., 35(1):65-73, Mar. 2006.

9. R. Fagin, P. G. Kolaitis, R. J. Miller, and L. Popa. Data exchange: semantics and query answering. Theoretical Computer Science, 336(1):89 - 124, 2005. Database Theory.

10. L. Galárraga, S. Razniewski, A. Amarilli, and F. M. Suchanek. Predicting Completeness in Knowledge Bases. In WSDM, pages 375-383, 2017.

11. G. Gottlob, G. Orsi, and A. Pieris. Ontological queries: Rewriting and optimization. In $I C D E$, pages $2-13,2011$.

12. G. Gottlob, G. Orsi, and A. Pieris. Query rewriting and optimization for ontological databases. CoRR, abs/1405.2848, 2014.

13. I. Ileana, B. Cautis, A. Deutsch, and Y. Katsis. Complete yet practical search for minimal query reformulations under constraints. In Proceedings of the 2014 ACM SIGMOD International Conference on Management of Data, SIGMOD '14, pages 1015-1026, New York, NY, USA, 2014. ACM.

14. D. S. Johnson and A. C. Klug. Testing containment of conjunctive queries under functional and inclusion dependencies. J. Comput. Syst. Sci., 28(1):167-189, 1984.

15. D. Lembo, M. Lenzerini, R. Rosati, M. Ruzzi, and D. F. Savo. Inconsistencytolerant query answering in ontology-based data access. Web Semantics: Science, Services and Agents on the World Wide Web, 33:3 - 29, 2015.

16. T. Lukasiewicz, M. V. Martinez, and G. I. Simari. Inconsistency handling in datalog+/- ontologies. In ECAI 2012 - 20th European Conference on Artificial Intelligence. Including Prestigious Applications of Artificial Intelligence (PAIS2012) System Demonstrations Track, Montpellier, France, August 27-31 , 2012, pages 558-563, 2012.

17. D. Maier, A. O. Mendelzon, and Y. Sagiv. Testing implications of data dependencies. ACM Trans. Database Syst., 4(4):455-469, Dec. 1979.

18. A. Poggi, D. Lembo, D. Calvanese, G. De Giacomo, M. Lenzerini, and R. Rosati. Linking data to ontologies. In Journal on data semantics X, pages 133-173. Springer, 2008.

19. A. Schwarte, P. Haase, K. Hose, R. Schenkel, and M. Schmidt. FedX: Optimization Techniques for Federated Query Processing on Linked Data. In ISWC, pages 601$616,2011$.

20. G. Weikum, J. Hoffart, and F. M. Suchanek. Ten years of knowledge harvesting: Lessons and challenges. IEEE Data Eng. Bull., 39(3):41-50, 2016. 